\section{Military Technical College Kobry El-Kobbah, Cairo, Egypt.}

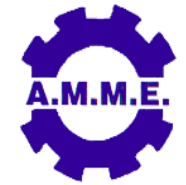

$18^{\text {th }}$ International Conference on Applied Mechanics and Mechanical Engineering.

\title{
MODELING LIGHTNING STRIKE DAMAGE IN CFRP COMPOSITES
}

\author{
Lin $\mathrm{Ye}^{\star}$
}

\begin{abstract}
Carbon Fiber Reinforced Plastics (CFRP) composites are widely used in modern airplanes to replace conventional metal alloys due to their high strength, high stiffness and light weight. Compared to their metallic counterparts, CFRP composites are vulnerable to lightning strike, and thus Lightning Strike Protection (LSP) system is required. In this work, a coupled thermal-electrical Finite Element (FE) model was used to predict thermal damage in CFRP composites with and without LSP systems during a lightning strike. Pyrolysis of polymer matrices and dielectric breakdown of the insulating layer in LSP system were considered. In addition, lightning strike induced mechanical damage in CFRP composites with a full thermal-electrical protection was determined using a mechanistic FE analysis together with a plasma expansion model, considering different failure phenomena, such as intralaminar damage and interlaminar delamination.
\end{abstract}

\section{KEY WORDS}

Lightning Strike Damage, CFRP Composites, FE Modeling, Thermal-electrical transition, Plasma expansion

* Professor, School of Aerospace, Mechanical and Mechatronic Engineering, University of Sydney, NSW, Australia. Email: lin.ye@sydney.edu.au 


\section{ABBREVIATIONS AND NOMENCLATURE}

$\begin{array}{ll}\text { CFRP } & \text { Carbon fibre reinforced plastics } \\ \text { LSP } & \text { Lightning strike protection } \\ \text { FE } & \text { Finite element } \\ \text { CE } & \text { Conductive CNTs/epoxy layer } \\ \text { NE } & \text { Non-conductive neat epoxy layer } \\ \text { IE } & \text { Insulating boron nitride modified epoxy layer } \\ \text { DI } & \text { Damage index } \\ I_{p e a k} & \text { Peak current } \\ \alpha, \beta, k & \text { Lightning current waveform constants } \\ L(t) & \text { Gap distance } \\ P(t) & \text { Lightning-induced pressure } \\ F & \text { Fraction of the internal energy to the thermal energy } \\ Q(t) & \text { The absorbed energy per unit area } \\ \mu_{p} & \text { The mass per unit area of protecting layer } \\ \mu_{c} & \text { The mass per unit area of protecting layer }\end{array}$

\section{INTRODUCTION}

Carbon fiber reinforced plastics (CFRP) composites are increasingly used to replace conventional metal alloys in aerospace and transportation industries due to their high strength-weight ratio and high stiffness-weight ratio in performance. However, the use of semi-conductive CFRP composites in a modern aeroplane presents a lightning strike threat to the flight safety. Compared to their metallic counterparts, CFRP composites exhibit a much lower electrical conductivity, which may not readily conduct away a high lightning current. Thus, the lightning may cause intensive resistive heating in the CFRP composites, leading to severe ablation damage such as pyrolysis of polymer matrices and burning of carbon fibers. To prevent the damage to a CFRP composite, a lightning strike protection (LSP) system, such as metal mesh with an insulating fibreglass layer, has been attached to the composite surface to conduct away the high lightning current. The thermal damage was sometimes observed in the composites with an LSP system [1] after lightning strike due to the poor design of the LSP system. Meanwhile, a significant amount of lightning energy is delivered quickly onto the materials, causing the ionised channel to expand. High-temperature plasma expansion creates a pressure pulse on the CFRP composites, creating more damage in structures.

Lightning-composite interaction is difficult to apprehend due to the lack of rigorous studies in this area. It is also hard and expensive to experimentally simulate lightning strikes in a laboratory without appropriate experimental set-ups and advanced equipment. Numerical modelling is one of the most effective methods to predict lightning strike damage in CFRP composites with significantly lower costs than the actual lightning strike experiments. This work provides a short overview of numerical modelling of lightning strike damage in CFRP laminates with and without LSP systems. The thermal-electrical transition induced thermal damage and the plasma expansion induced mechanical damage in the CFRP composite during a lightning strike are addressed. 


\section{LIGHTNING CURRENT WAVEFORM}

A lighting strike is associated with a high lightning current which typically consists of four stokes, i.e. initial stroke, intermediate stroke, continuous stroke and restrike. At the laboratory, the current generator [2] is sometimes applied to create current waveforms to simulate the lightning strike in the natural environment. MIL-STD-464 standard [3] divides the lightning waveform into four components as shown in Fig. 1a.

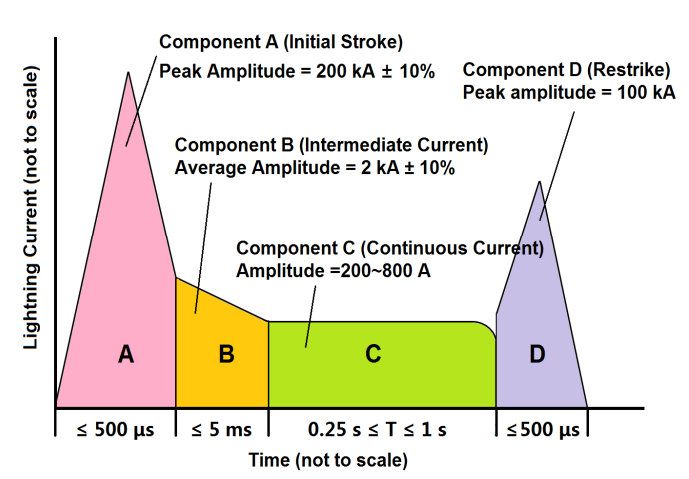

(a)

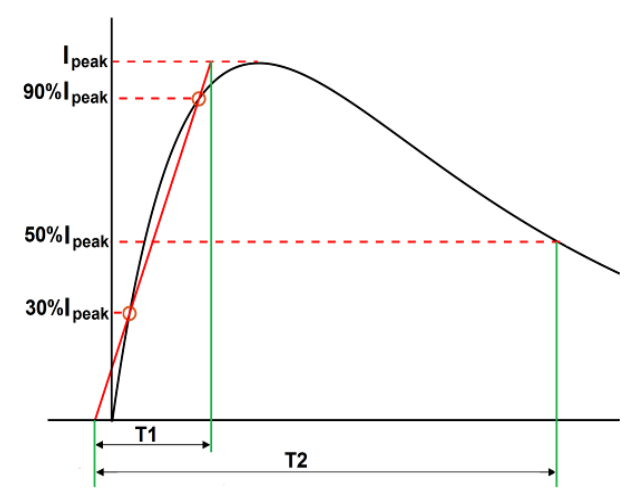

(b)

Fig.1. Schematic lightning current waveform.

It is seen that Component $A$ represents an initial stroke with a peak magnitude of 200 $\mathrm{kA}$, much higher than those of Component $\mathrm{B}$ and $\mathrm{C}$. Component $\mathrm{D}$ is a typical lightning restrike with a peak magnitude of $100 \mathrm{kA}$ with duration of $500 \mu \mathrm{s}$. Component $A$ and $D$ are two high-magnitude currents and thus are the focus of the study. Component $A$ and $D$ can be approximated by double exponential waveforms [3] as follows,

$$
I(t)=k I_{p e a k}\left(e^{-\alpha t}-e^{-\beta t}\right)
$$

where $I_{\text {peak }}$ is the peak magnitude of the current. $\alpha, \beta, k$ are the constants. The definition of the lightning current provides the input for the following FE modelling.

\section{THERMAL-ELECTRICAL TRANSITION}

For characterizing thermal damage in CFRP laminate without protection, a coupled thermal-electrical FE model [4] was developed considering pyrolysis of polymer matrices under Joule heating. The CFRP composite with a lay-up sequence of [45/0/$45 / 90]_{2 S}$ has the dimensions of $150 \mathrm{~mm} \times 100 \mathrm{~mm} \times 3.2 \mathrm{~mm}$. The boundary condition of the FE model was comparable with that of the lighting strike experiment [1]. A concentrated lighting current was applied onto the centre of the composite with a magnitude of $40 \mathrm{kA}$ and $100 \mathrm{kA}$, respectively. The zero potential was applied on two ends of the CFRP laminates to simulate the grounding effect. Heat radiated from all the free surfaces of the composite, and the ambient temperature was set to be $25{ }^{\circ} \mathrm{C}$. The virtual latent heat was introduced in the model to limit the maximum temperature to $3316^{\circ} \mathrm{C}$.

After applying a $40 \mathrm{kA}$ lightning current, thermal damage index (DI) of the laminate is illustrated in Fig. 2a. $45^{\circ}$ stripe-shaped damage is observed, which is consistent with experimental observation [1], as shown in Fig. 2d. The front ply has a fiber orientation 

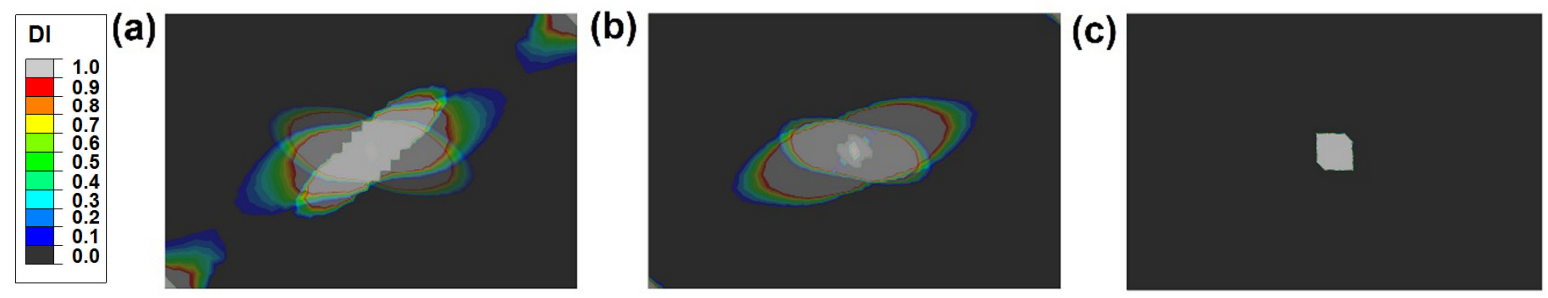

(d)

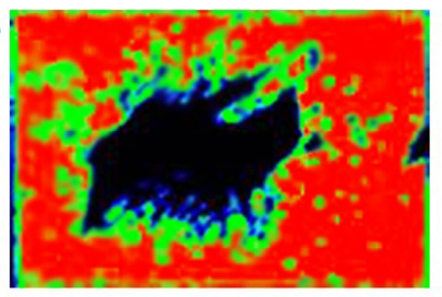

(e)
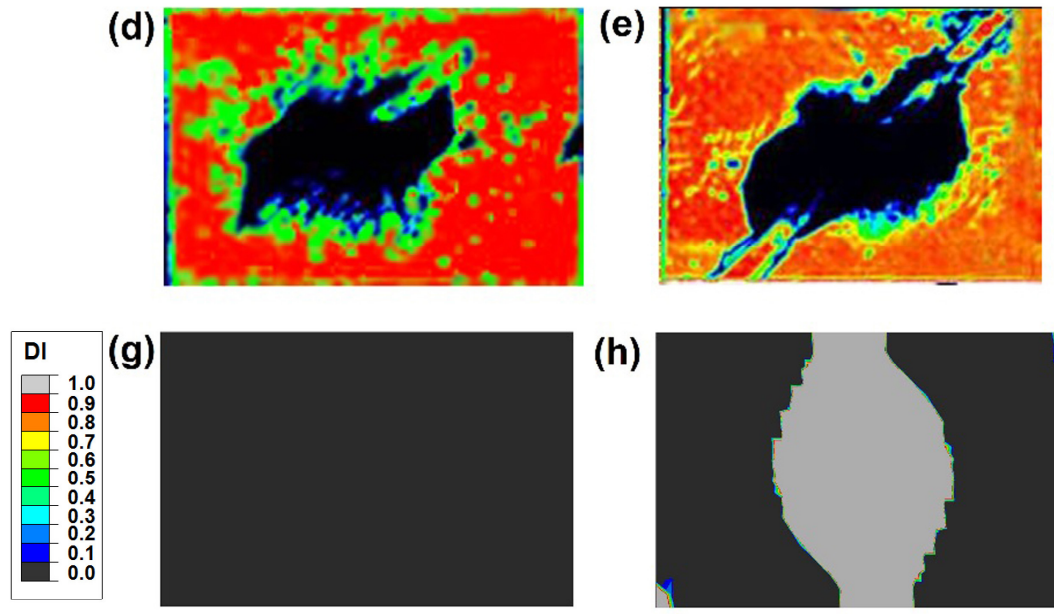

(j)

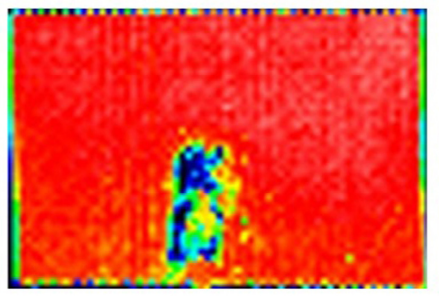

(h)

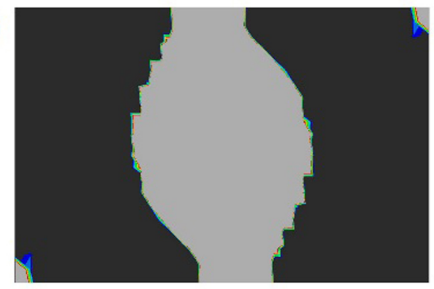

(k)

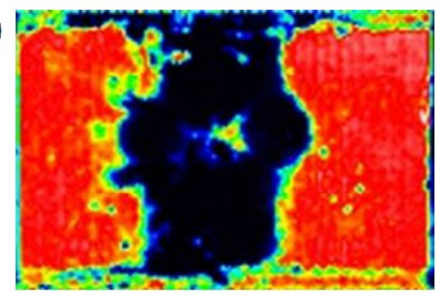

(f)

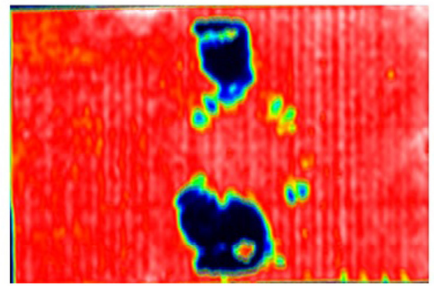

(i)

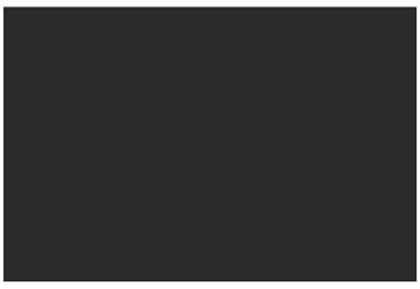

(I)

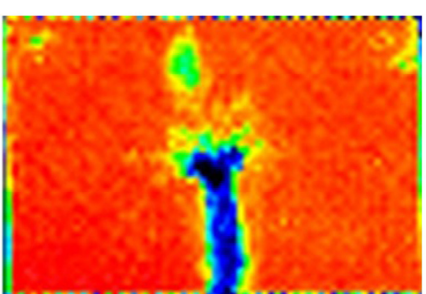

Fig. 2. Thermal damage by the FE model on the specimen of (a) CFRP-40 kA (b) BP/CEP -40 kA (c) BP/NEP-40 kA (g) BP/IEP-40 kA (h) BP/NEP-100 kA (i) BP/IEP-100 kA; and C-scan results of (d) CFRP-40 kA (e) BP/CEP-40 kA (f) BP-/NEP-40 kA (j) BP/IEP-40 kA by FE model, (k) BP/NEP-100 kA (l) BP/IEP-100 kA [4].

of $45^{\circ}$. As the lowest electrical resistivity happens in the fibre direction, most of the currents flow along the fibers, causing the stripe-shaped damage in the laminate. By examining the damage area and depth in Table 1, it is found that the damage area and depth predicted by our FE model agree well with those obtained by the experiment [1]. The deviation of the damage area and depth between the FE model results and experiment is approximately $3 \%$ and $22 \%$, respectively. These errors may be resulted by the introduction of the virtual latent heat. In the experiment, ply lift or delamination were observed, indicating a partly release of absorbed energy. However, in our FE model, it is assumed that the stored energy is absorbed by the virtual latent heat after the carbon fiber sublimation. The mechanical damage is neglected here.

In this work, buckypaper and adhesives were used as an LSP system as shown in Fig. 3. Buckypaper was used as a conductive layer, and adhesives were used as insulating layers. To examine the effect of adhesives, three types of adhesives, a conductive CNTs/epoxy layer (CE), a non-conductive neat epoxy layer (NE), and an 
Table 1. Summary of damage area and depth from FE and experiments [4]

\begin{tabular}{|c|c|c|c|c|}
\hline Specimen & $\begin{array}{c}\text { Damage } \\
\text { area }\left(\mathrm{mm}^{2}\right) \\
\mathrm{FE}\end{array}$ & $\begin{array}{c}\text { Damage area } \\
\left(\mathrm{mm}^{2}\right) \\
\text { Experiment }\end{array}$ & $\begin{array}{c}\text { Damage depth } \\
(\mathrm{mm}) \\
\mathrm{FE}\end{array}$ & $\begin{array}{c}\text { Damage depth } \\
(\mathrm{mm}) \\
\text { Experiment }\end{array}$ \\
\hline CFRP & 3237 & $\sim 3380$ & 0.7 & 0.9 \\
\hline BP/CEP 40 kA & 2335 & $\sim 4650$ & 0.7 & 0.8 \\
\hline BP/NEP-40 kA & 169 & $\sim 940$ & 0.1 & $\sim 0$ \\
\hline BP/IEP-40 kA & 0 & 0 & 0 & 0 \\
\hline BP/NEP-100 kA & 5015 & $\sim 4460$ & 0.1 & $\sim 0$ \\
\hline BP/IEP-100 kA & 0 & 0 & 0 & 0 \\
\hline
\end{tabular}

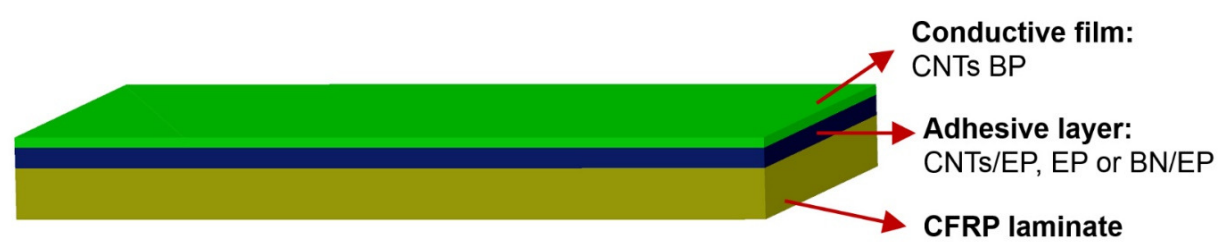

Fig. 3. Schematic of CFRP laminates with LSP system [4].

insulating boron nitride modified epoxy layer (IE) were utilised here. The adhesive layer may experience dielectric breakdown during a lightning strike. Thus, an ABAQUS User subroutine was incorporated into the FE model to address this phenomenon by comparing the breakdown strength with the electrical potential gradient.

Here, the damage in the CFRP composite with different LSP systems is predicted using the FE model. Fig. $2 \mathrm{~b}$ displays thermal damage in the CFRP laminate with $\mathrm{a}$ conductive adhesive LSP system (BP/CEP) under a $40 \mathrm{kA}$ current. It can be seen that the shape of damage is consistent with the experimental observation shown in Fig. 2e. The damage depth in the experiment is well predicated with an error of $12.5 \%$, whereas the damage area by the FE model is smaller than that in the experiment with a deviation of $49.7 \%$ because the ply lift damage is neglected in our FE model. In the thickness direction, Joule heating has more contribution to the determination of the damage. Hence, the predicted damage depth agrees well with the experimental result. In addition, it is predicted that dielectric breakdown occurs at the centre of the adhesive layer, facilitating the currents flowing in the thickness direction, causing the damage in the CFRP composite. BP/CEP is not a good candidate for an LSP system.

The effectiveness of the LSP with a non-conductive epoxy is also examined. Fig. 2c shows that the damage area in the specimen of BP/NEP is significantly reduced. Table 1 shows the damage area and depth are $169 \mathrm{~mm}^{2}$ and $0.1 \mathrm{~mm}$, respectively. However, compared with the experimental result in Fig. 2f, the damage shape is entirely different. In the experiment, it is found that the damage does not locate at the centre of the specimen, which suggests a surface flashover happen from the lightning attachment point to another point. In our FE model, this phenomenon is not considered, which results in the difference in the damage shape. We also examine 
the breakdown status of the NEP; it is observed that dielectric breakdown also happens in the NEP.

Figure $2 \mathrm{~g}$ and Table 1 show thermal damage in the specimen of BP/CEP from the FE model. It is seen that the damage area and depth are both zero, which is comparable with the experimental results. The CFRP is well protected by this type of LSP system. There are three reasons for BP/IEP to be a valid candidate for an LSP system. First, the buckypaper can conduct away most of the lightning currents due to its high conductivity. Second, the insulating layer can prevent the currents flowing in the thickness direction owing to its high electric resistivity. Third, the high breakdown strength of the insulating layer can provide reliable protection for the CFRP composite.

Next, the magnitude of the applied current increases to $100 \mathrm{kA}$. In the previous analysis it was shown that BP/NEP and BP/IEP can be used as suitable candidates for LSP systems. We further examine the performance of these two types of LSP system when a high magnitude current (i.e. $100 \mathrm{kA}$ ) is applied. Fig. $2 \mathrm{~h}$ shows the thermal damage in the specimen of BP/NEP. It is shown that almost one-third of the specimen surface area is damage due to the high current. Table 2 shows that the damage area is much larger than that in Fig. 2c. However, the predicted damage depth is only $0.1 \mathrm{~mm}$, which suggests that the damage be confined to the front layer of the laminates. The results of the damage shape, damage area and depth are in good agreement with the experimental data [1]. When a $100 \mathrm{kA}$ current is applied, dielectric breakdown occurs, and thus, the currents flow into the CFRP composites, which leads to damage in the CFRP. However, for the specimen of BP/IEP under a $100 \mathrm{kA}$ current, no damage is found as shown in Fig. $2 \mathrm{i}$ and Table 1, which is comparable to the experimental observation [1]. It is evident that the advanced LSP system with an insulating adhesive layer can protect the CFRP composite well even under striking of a high magnitude lightning current.

\section{SURFACE PLASMA EXPANSION}

When a high-energy lightning strikes onto a CFRP composite laminate, the surface is vaporized in a short time and is transformed into plasma by ionisation. Hightemperature plasma creates a pressure pulse onto the laminate. Accordingly, a shock wave is generated inside the composite, leading to mechanical damage to the laminate. The mechanical damage is more severe if the plasma is confined by the protecting layer on top of the laminates. Fabbro et al. [5] developed a onedimensional surface explosion model for estimating a high-energy laser-induced pressure pulse. This model can be extended to predict the pressure pulse during a lightning strike [6]. When a cylindrical high-energy lightning is applied, the plasma expands onto the protected CFRP laminate. The relation between the timedependent pressure $P(\mathrm{t})$ and the gap distance $L(\mathrm{t})$ can be determined by the following equation,

$$
\frac{d^{2} L(t)}{d t^{2}}=\left(\frac{1}{\mu_{p}}+\frac{1}{\mu_{c}}\right) P(t)
$$

where $\mu_{p}$ and $\mu_{c}$ are the mass per unit area of protecting layer and CFRP laminate. 
According to the energy conservation formula, the power per unit area consists of the internal energy and the work by the pressure pulse,

$$
Q(t)=P(t) \frac{d L}{d t}+\frac{d}{d t}\left(\frac{3}{2 F} P L\right)
$$

where $F$ is a fraction of the internal energy. $Q(\mathrm{t})$ can be simplified as:

$$
Q(t) \approx \frac{10 I(t)}{\pi R(t)^{2}}
$$

Subsequently, the solution of the pressure pulse can be determined by numerically solving the Eqns. (1) - (3). Prior to the FE analysis, the lightning-induced pressure magnitude is obtained by the surface explosion model described in Eqns. (2) - (4). Figure $4 \mathrm{a}$ shows the predicted pressure pulse caused by a lightning current waveform $\mathrm{T} 1 / \mathrm{T} 2=2.6 \mu \mathrm{s} / 50 \mu \mathrm{s}$ ( $\mathrm{T} 1$ is front time and $\mathrm{T} 2$ is tail time). It is found that the pressure waveforms are similar when different peak currents are utilized. The predicted pressure magnitude reaches its peak in a very short time (less than $1 \mu \mathrm{s}$ ). After that, the pressure drops back to zero in $\sim 100 \mu \mathrm{s}$. The $200 \mathrm{kA}$ current (Component A) induces the highest pressure magnitude of $\sim 158 \mathrm{MPa}$, which is adopted to investigate the mechanical damage in the fully protected CFRP laminate.

An explicit FE model describing the mechanical damage of a CFRP laminate of full electrical-thermal protection was developed using ABAQUS. In this case, transition of lightning electricity and Joule heating are not considered. A laminate with a lay-up sequence of $\left[45^{\circ} / 0^{\circ} /-45^{\circ} / 90^{\circ}\right]_{s}$ as an 8 -ply quasi-isotropic laminate was considered as a case study. The dimension of the laminate is $450 \mathrm{~mm} \times 450 \mathrm{~mm} \times 1.5 \mathrm{~mm}$, and fixed constraints were applied on four edges of the laminate. A cylindrical pressure pulse was applied to the centre of the laminate. Progressively intra-laminar damage model and interlaminar delamination model were discussed in detail in our previous work [6]. We apply the pressure pulse predicted as above in the FE model. The deflection response of the CFRP laminate is examined. On the bottom surface, the centre and other four sampling points (of a distance D) located in the same direction with an interval of $24 \mathrm{~mm}$ are chosen. In Figure $4 \mathrm{~b}$, the greatest deflection happens in the centre at $\sim 48 \mu \mathrm{s}$, with a value of $\sim 9.72 \mathrm{~mm}$. After that, the deflection gradually decreases with time. For the sampling location points away from the centre, a pressure pulse-induced shock wave propagation is found. The responses of the four sampling locations are delayed at the same interval, and their peak deflections decrease with the distance from the centre. The deflection caused by the pressure pulse is significant, and the shock wave propagation may induce damage in the laminate.

Next, the intra-laminar damage and interlaminar delamination of the CFRP laminate are obtained. It is observed that damage primarily happens in the bottom ply. In order to determine the failure modes and damage evolution, the damage variables are plotted at an interval of $50-500 \mu$ s as shown in Figure 5 . It can be seen that the damage variables show a $45^{\circ}$ orientation due to the fiber direction in this ply is $45^{\circ}$. At $50 \mu \mathrm{s}$, a few elements were fully damage and removed as the corresponding damage variables for compression reach one. Fiber damage in tension is not found, and therefore, the damage is mainly caused by compression. Meanwhile, the matrix 


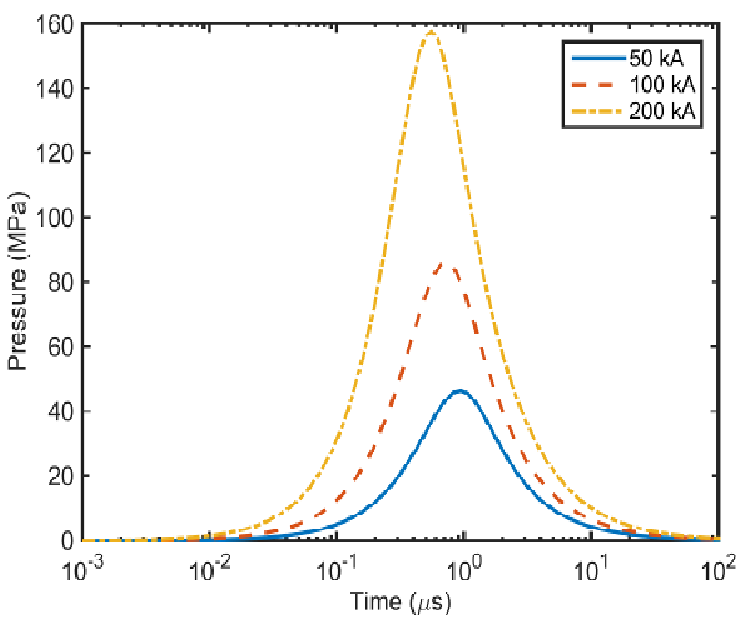

(a)

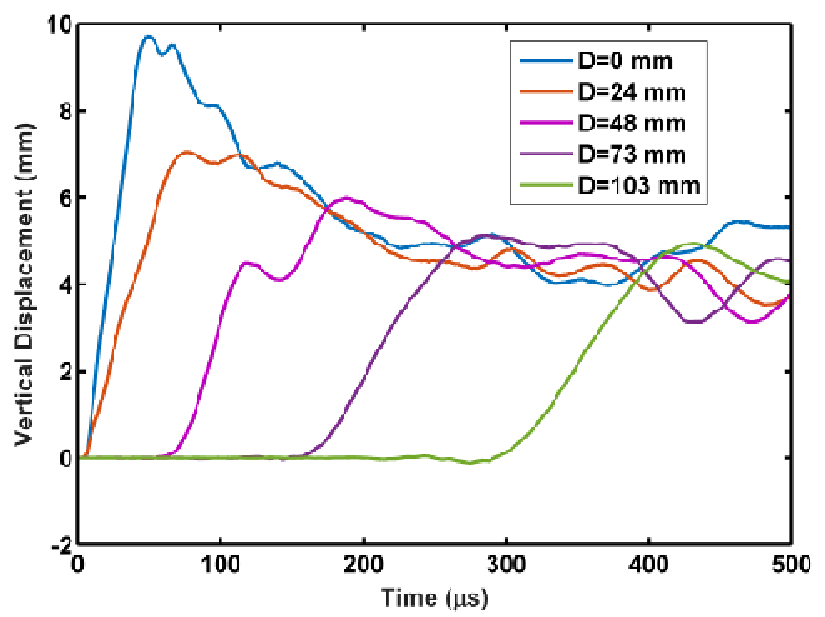

(b)

Fig. 4. (a) Pressure pulse by surface plasma expansion model and (b) vertical displacement responses at different sampling locations by the FE model
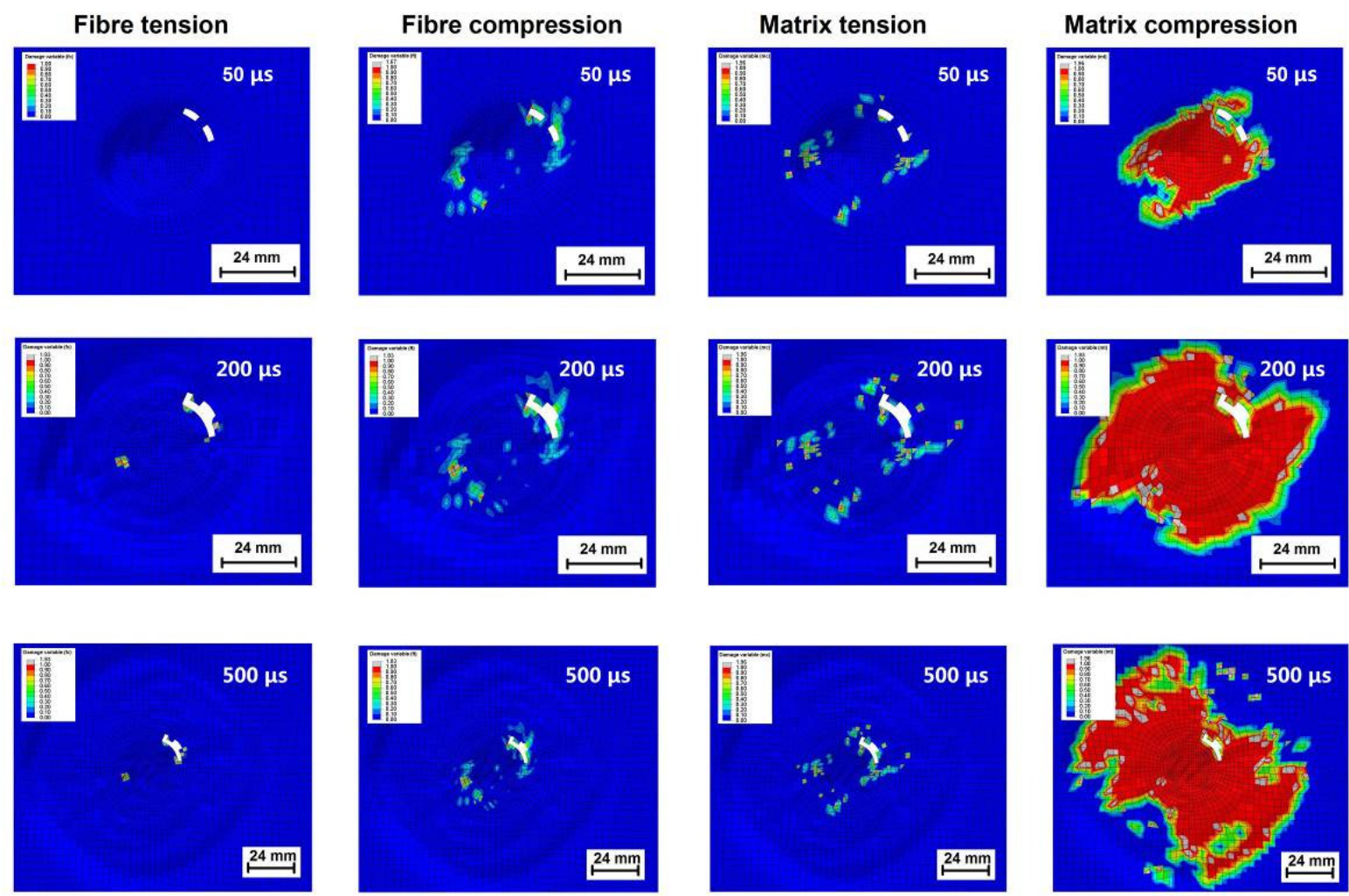

Fig. 5. Evolution of damage variables in CFRP laminates with time [6].

damage is severe in the bottom ply. As time increases, more elements are damage and removed due to the shock wave propagation, e.g. at $200 \mu$ s and $500 \mu \mathrm{s}$. In addition, the area of matrix damage also increases. 


\section{CONCLUSIONS}

(1) An FE model was modified to incorporate the dielectric breakdown behavior of the LSP system, addressing the effects of dielectric breakdown.

(2) The shape, area and depth of damage predicted by the modified FE model were comparable with the experimental data, validating the feasibility of the current FE model.

(3) The use of a buckypaper/insulating layer as an advanced LSP system could successfully protect the composites from lightning strike damage owing to the high dielectric strength and low conductivity of the insulating layer.

(4) An FE model with a surface plasma explosion model has been developed to predict the mechanical damage in CFRP laminates with full electrical-thermal protection, caused by a lightning strike. The peak deflection was $9.72 \mathrm{~mm}$ for a lightning strike with a peak current of $200 \mathrm{kA}$.

\section{REFERENCES}

[1] J.H. Han, H. Zhang, M.J. Chen, D. Wang, Q. Liu, Q.L. Wu, Z. Zhang, "The combination of carbon nanotube buckypaper and insulating adhesive for lightning strike protection of the carbon fiber/epoxy laminates", Carbon, Vol. 94, pp. 101-113, (2015)

[2] M.I. Baranov, G.M. Koliushko, V.I. Kravchenko, O.S. Nedzel'skii, V.N. Dnyshchenko, "A current generator of the artificial lightning for full-scale tests of engineering objects", Instrum. Exp. Tech., Vol. 51, No. 3, pp. 401-405. (2008)

[3] "Military Standard. Lightning qualification test techniques for aerospace vehicles and hardware", MIL-STD-1757, (1983)

[4] K.K. Fu, L. Ye, L. Chang, C.H. Yang, Z. Zhang, "Modelling of lightning strike damage to CFRP composites with an advanced protection system. Part I: Thermal-electrical transition", Compos. Struct., Vol. 165, pp.83-90. (2017)

[5] R. Fabbro, J. Fournier, P. Ballard, D. Devaux, J. Virmont, "Physical study of laser-produced plasma in confined geometry", J. Appl. Phys., Vol. 68, pp. 775784, (1990)

[6] K.K. Fu, L. Ye, L. Chang, C.H. Yang, "Modelling of lighting strike damage of CFRP laminates caused by sudden surface plasma explosion", 21st International Conference on Composite Materials, Xi'an, China, (2017) 